\title{
A Novel DOA Estimation Based on State Space Balance Method and Its Performance Analysis
}

\author{
Hong Xiang ${ }^{1, a}$, Jun Wang ${ }^{2, b^{*}}$ Zhaotao Qin ${ }^{3, c}$ and Hai Jiang ${ }^{4, d}$ \\ ${ }^{1,2,3}$ School of electronic information engineering, Beihang University, Beijing, China \\ ${ }^{4}$ Key laboratory of space physics, Beijing, China \\ axianghong@buaa.edu.cn, 'wangDSP203@163.com, 'qinzhaotao2008@163.com, \\ dbradley0226@163.com
}

\begin{abstract}
Keywords: DOA estimation, state-space balance method, single snapshot, Hankel matrix. Abstract. Considering the high speed space targets, the real time processing is required with only a few snapshots. Meanwhile, the algorithm based on state space method performs well in the parameter estimation. In this work, we present a novel Direction of Arrival (DOA) estimation based on state space balance method. Firstly, two hankel matrixes based on the received data are constructed. Then the singular value decomposition of the hankel matrix is used to obtain the signal subspace and singular value to estimate the DOA. This proposed algorithm acquires a good performance and a quite efficiency calculation compared with MUSIC algorithm and ESPRIT algorithm. At the same time, the proposed method has the ability to estimate the DOAs of multi-targets by using only a single snapshot. Simulation studies show that the proposed algorithm is correct and useful.
\end{abstract}

\section{Introduction}

Direction of Arrival (DOA) estimation is one of the main research directions in array signal processing, which plays an important role in radar, sonar, radio communication and radio astronomy etc. The earliest DOA algorithm based on array processing is the conventional beam forming (CBF) method [1]. However, this method is unable to break through the Rayleigh limit and cannot distinguish targets from a beam width. The research of Modern super resolution direction finding technology is developed well because of its super resolution ability. Domestic and foreign scholars have put forward linear prediction algorithm [2], the subspace algorithm [3-6], subspace fitting algorithm [7, 8] and so on. In order to get the super resolution DOA estimation, the subspace algorithm uses the orthogonal properties between the signal subspace and the noise subspace to construct the spatial spectrum peak. The subspace algorithm mainly includes the Multiple Signal Classification (MUSIC) algorithm based the noise subspace and Estimation of Signal Parameters via Rotational Invariance Techniques (ESPRIT) algorithm based the signal subspace. MUSIC algorithm has the better performance than ESPRIT algorithm while ESPRIT algorithm owns lower computation than MUSIC algorithm. However, both algorithms thought that the covariance matrix contains the information of targets and background interference. They use the covariance matrix of the array received signal to realise the DOA estimation. However, the method that directing use of received data to get the information of targets and back ground interference rarely appear in the relevant papers. In fact, covariance matrix and direct use received data both have the ability to acquire the information while direct use received data will increase the dimension of the construct matrix if the samples of snapshot increase which analyses in paper [9]. However, aiming at the high speed space targets, the real time processing is required with only a few snapshots. Therefore, the disadvantage of matrix dimension increased can be ignored. We can adopt the direct received data to estimate the DOA. In paper [10], a state-space approach is used to extract the UWB scattering center of moving target and the precision of estimation performance is very excellent by using the frequency domain data.

In this paper, we present a novel algorithm for DOA estimation based on the state-space balance method. Firstly, two hankel matrixes based on the received data are constructed. Then the singular value decomposition of the hankel matrix is used to obtain the signal subspace and singular value to estimate the DOA. This proposed algorithm acquires a good performance on the estimation error and 
a lower calculation compared with that of MUSIC algorithm while acquire a better performance on the estimation error and a quite efficiency calculation compared with that of ESPRIT algorithm. At the same time, the proposed method has the ability to estimate the DOAs of multi-targets by using only a single snapshot. Simulation studies show that the proposed algorithm is correct and useful.

\section{Singal Model}

In order to use state-space method to estimate the DOA, this paper construct a uniform linear array with the array element spacing $d \leq \lambda / 2$, as shown in Fig. $1 . \lambda$ is the received signal wavelength.

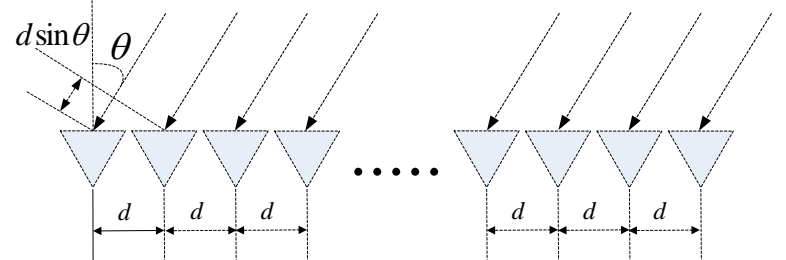

Fig. 1 Uniform Linear Array with the Incident Signal

Assume $K$ far-field uncorrelated narrowband plane waves $s_{i}(t) \quad(i=1,2, \cdots, K)$ simultaneously incident on the uniform linear array, with $M$ array element. $\theta_{i}(i=1,2, \cdots, K)$ is the DOA of the incident signal. The noise is the additive white Gaussian noise (AWGN) with zero means. The relationship between the noise and signal is independent of each other. The noise of each array element is no correlation. Without considering the effect of mutual coupling between channels and other factors, taken the first array element as a reference element, the time delay $\tau_{l i}$ of the $i$ th incident signal to the $l$ th array element is given by

$$
\tau_{l i}=\frac{(l-1) d \sin \theta_{i}}{c}(i=1,2, \cdots, K)
$$

For the far-field signal s, the following equation can be established as [11]

$$
s_{i}\left(t-\tau_{l i}\right) \approx s_{i}(t) \exp \left(-j \omega_{0} \tau_{l i}\right)
$$

where $\omega_{0}=2 \pi f=2 \pi c / \lambda$ is the frequency of the received signal. Therefore, we can get the received signal of the $l$ th array element from equation (1) and equation (2) that

$$
x_{l}(t)=\sum_{i=1}^{K} g_{l i} s_{i}(t) \exp \left(-j \omega_{0} \frac{(l-1) d \sin \theta_{i}}{c}\right)+n_{l}(t)
$$

where $g_{l i}$ stand for the gain of the $l$ th array element to the $i$ th received signal. $n_{l}(t)$ is the noise component of the $l$ th array element. The equation (3) can be written as a matrix as follows

$$
x_{l}(\mathrm{t})=C \bullet A^{l-1} \bullet B+n_{l}(t)
$$

where $C=\left[\begin{array}{llll}g_{l 1} & g_{l 2} & \cdots & g_{l K}\end{array}\right]$ is the gain vector while $B=\left[\begin{array}{llll}s_{1}(t) & s_{2}(t) & \cdots & s_{K}(t)\end{array}\right]^{\mathrm{T}}$ is the signal vector. $(\cdot)^{T}$ is transpose. $A$ is a diagonal matrix which can be expressed as follows

$$
A=\left[\begin{array}{cccc}
\mathrm{e}^{-j \omega_{0} d \sin \theta_{1}} & 0 & 0 & 0 \\
0 & \mathrm{e}^{-j \omega_{0} d \sin \theta_{2}} & 0 & 0 \\
0 & 0 & \ddots & 0 \\
0 & 0 & 0 & \mathrm{e}^{-j \omega_{0} d \sin \theta_{K}}
\end{array}\right]
$$

From equation (5), if the phase of the diagonal element of matrix $A$ is estimated, the DOA of $\theta_{i}$ can be acquired. 


\section{State-space balance method}

As is shown in paper [10], hankel matrix is constructed to estimate the information of the diagonal matrix by using the state-space observation method. This paper will use the state-space balance method to estimate the DOA of incident signal.

Hankel matrix $H_{0}$ can be structured by the received data $x_{l}(t)$ from the first $M-1$ array elements. The expression is given by

$$
H_{0}=\left[\begin{array}{cccc}
x_{1}(\mathrm{t}) & x_{2}(\mathrm{t}) & \cdots & x_{M-M R}(\mathrm{t}) \\
x_{2}(\mathrm{t}) & x_{3}(\mathrm{t}) & & \vdots \\
\vdots & & \ddots & \\
x_{M R}(\mathrm{t}) & \cdots & & x_{M-1}(\mathrm{t})
\end{array}\right]
$$

Hankel matrix $H_{1}$ can be structured by the received data $x_{l}(t)$ from the last $M-1$ array elements. The expression is given by

$$
H_{1}=\left[\begin{array}{cccc}
x_{2}(\mathrm{t}) & x_{3}(\mathrm{t}) & \cdots & x_{M-M R+1}(\mathrm{t}) \\
x_{3}(\mathrm{t}) & x_{4}(\mathrm{t}) & & \vdots \\
\vdots & & \ddots & \\
x_{M R+1}(\mathrm{t}) & \cdots & & x_{M}(\mathrm{t})
\end{array}\right]
$$

In order to get the best estimation accuracy of DOA, the row number of the hankel matrix set as follows

$$
M R=\text { floor }((M-1) * 2 / 3)+1
$$

where floor $(\cdot)$ is the rounding function.

According to equation (4) without considering the noise component, equation (7) and equation (8) can be written as

$$
\begin{aligned}
& H_{0}=\underbrace{\left[\begin{array}{c}
C A^{0} \\
C A^{1} \\
\vdots \\
C A^{M R-1}
\end{array}\right]}_{\Omega} \cdot \underbrace{\left[\begin{array}{llll}
A^{0} B & A^{1} B & \cdots & A^{M-M R-1} B
\end{array}\right]} \\
& H_{1}=\underbrace{\left[\begin{array}{c}
C A^{0} \\
C A^{1} \\
\vdots \\
C A^{M R-1}
\end{array}\right]}_{\Omega} \cdot A \cdot \underbrace{A \cdot\left[\begin{array}{llll}
A^{0} B & A^{1} B & \cdots & A^{M-M R-1} B
\end{array}\right]}_{\Theta}
\end{aligned}
$$

From equation (9) and equation (10), we can find the relationship between hankel matrix $H_{0}$ and hankel matrix $H_{1}$. Matrix $\Omega$ can be described as the observer matrix while matrix $\Theta$ can be described as the control matrix. In order to get the observer matrix $\Omega$ and the control matrix $\Theta$, the singular value decomposition of hankel matrix $H_{0}$ is acquired as follows

$$
H_{0}=U \Sigma V^{H}
$$

where matrix $\Sigma$ contains the singular values of matrix $H_{0}$, while matrix $U$ and matrix $V$ contain the corresponding singular vectors. Considering the signal part and the noise part, we can divide the matrix $H_{0}$ as the following equation given by

$$
H_{0}=\left[\begin{array}{ll}
U_{s} & U_{n}
\end{array}\right]\left[\begin{array}{cc}
\Sigma_{s} & 0 \\
0 & \Sigma_{n}
\end{array}\right]\left[\begin{array}{ll}
V_{s}^{H} & V_{n}^{H}
\end{array}\right]
$$


where $\Sigma_{s}$ stands for the larger singular values correspond to the signal part while $\Sigma_{n}$ stands for the smaller singular values correspond to the noise part. $U_{s}$ and $V_{s}^{H}$ stand for the signal vector while $U_{n}$ and $V_{n}^{H}$ stand for the noise vector. Using Akaike information criterion (AIC) [12,13] or minimum description length (MDL) $[14,15]$, we can effectively estimate the number of the $K$ incident signals and get the accurate signal vector.

Using the estimated number of the incident signals, we can get the $K$ order hankel matrix as shown below

$$
\hat{H}_{0}=U_{1: K} \Sigma_{1: K, 1: K} V_{1: K}^{H}=\hat{\Omega} \bullet \hat{\Theta}
$$

where $U_{1: K}$ is the top $K$ columns of matrix $U ; \Sigma_{1: K, 1: K}$ is the $K$ order diagonal matrix correspond to the $K$ singular values of matrix $\Sigma$ about the signal part. $V_{1: K}^{H}$ is the top $K$ columns of matrix $V^{H}$. Then, observer matrix $\hat{\Omega}$ and control matrix $\hat{\Theta}$ can be calculated as follows

$$
\begin{aligned}
& \hat{\Omega}=U_{1: K} \sqrt{\sum_{1: K, 1: K}} \\
& \hat{\Theta}=\sqrt{\sum_{1: K, 1: K}} V_{1: K}^{H}
\end{aligned}
$$

According to equation 10, diagonal matrix $\hat{A}$ can be estimated as follows

$$
\hat{\mathrm{A}}=\hat{\Omega}^{+} \bullet H_{1} \bullet \hat{\Theta}^{+}
$$

where $(\cdot)^{+}$stands for the generalized inverse.

Define $\lambda_{i}(i=1,2, \cdots, K)$ as the eigenvalue of matrix $\hat{\mathrm{A}}$, the estimation of DOA can be calculated by the following equation

$$
\sin \theta_{i}=\frac{1}{-j \omega_{0} d} \cdot \operatorname{angle}\left(\lambda_{i}\right),(i=1,2, \cdots, K)
$$

where angle $(\cdot)$ returns the phase angle.

In conclusion, the detailed steps for implementing the state-space balance method of DOA estimation algorithm can be expressed as follows

Step 1: Construct the hankel matrix $H_{0}$ and $H_{1}$ from the received data $x_{l}(t)$;

Step 2: Use the singular value decomposition of hankel matrix $H_{0}$ to obtain the signal subspace $U_{s}$ and singular value $\Sigma_{s}$.

Step 3: Use equation (14) and equation (15) to compute the observer matrix $\hat{\Omega}$ and the control matrix $\hat{\Theta}$

Step 4: Use equation (16) to obtain matrix Â;

Step 5: Use the eigenvalue of matrix $\hat{A}$ to estimate DOA.

\section{State-space balance method}

Through the simulation experiments, the state-space balance method in this paper will be compared with MUSIC algorithm and ESPRIT algorithm to verify the validity of the algorithm.

Experiment 1: verify the estimation error with the SNR

The DOA of incident signal situated at an angle $10^{\circ}$. The number of array elements is 50 and the array element spacing is $\lambda / 2$. The range of SNR is from $-20 d B$ to $40 d B$ where the step length is about $5 d B$. Five hundred independent Monte-Carlo experiments are tested at the each SNR condition. We study the estimation accuracy with the average root-mean-squared error (RMSE) against SNR, evaluated as

$$
R M S E=\sqrt{\frac{1}{M} \sum_{i=1}^{M}\left(\hat{\theta}_{i}-\theta\right)^{2}}
$$


where $M$ is the number of independent Monte-Carlo experiments, $\hat{\theta}_{i}$ is the single estimation DOA of the incident signal, $\theta$ is the real DOA of the incident signal. The simulation results are shown in Fig. 2. We can see from Fig. 2 that at the same SNR condition, the accuracy of the proposed method almost has the same performance with MUSIC algorithm and much better than ESPRIT algorithm. However, if we analysed the process of the construction hankel matrix $H_{0}$ and hankel matrix $H_{1}$, the order of the hankel matrix $H_{0}$ and $H_{1}$ is $2 \mathrm{M} / 3$. The proposed method acquires a better performance by sacrificing the array aperture.

Experiment 2: verify the estimation error with the snapshot

The DOA of incident signal situated at an angle $10^{\circ}$. The number of array elements is 50 and the array element spacing is $\lambda / 2$. SNR situated at $0 d B$. The range of snapshot is from 0 to 50 where the step length is about 5. Five hundred independent Monte-Carlo experiments are tested at each snapshot. The simulation results are shown in Fig. 3.

We can see from Fig. 3 that at the same snapshot condition, the accuracy of the proposed method almost has the same performance with MUSIC algorithm and much better than ESPRIT algorithm. The proposed algorithm can also satisfy the multiple sampling points.

Experiment 3: verify probability of resolution with SNR

The DOAs of incident signals situated at angle $10^{\circ}$ and $15^{\circ}$. The number of array elements is 50 and the array element spacing is $\lambda / 2$. The range of SNR is from $-2+-0 d B$ to $20 d B$ where the step length is about $1 d B$. The snapshot is single sample. Five hundred independent Monte-Carlo experiments are tested at each snapshot. The simulation results are shown in Fig. 4 . We can see from Fig. 4 that with only a snapshot, the proposed algorithm has a quite excellent ability to distinguish the DOAs of multi-targets.

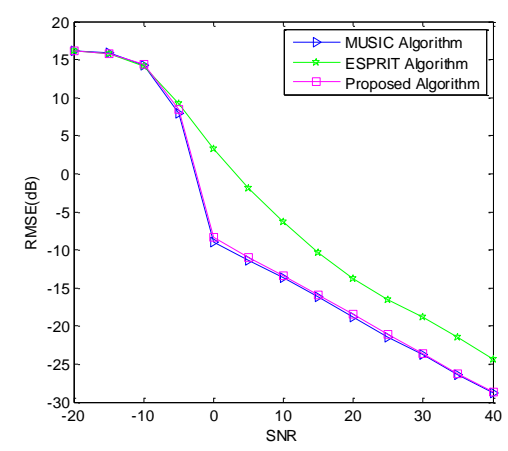

Fig. 2 RMSEs of angle estimation against SNR

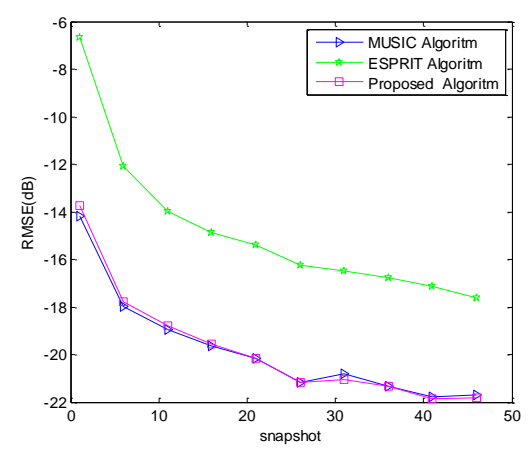

Fig. 3 RMSEs of angle estimation against snapshot

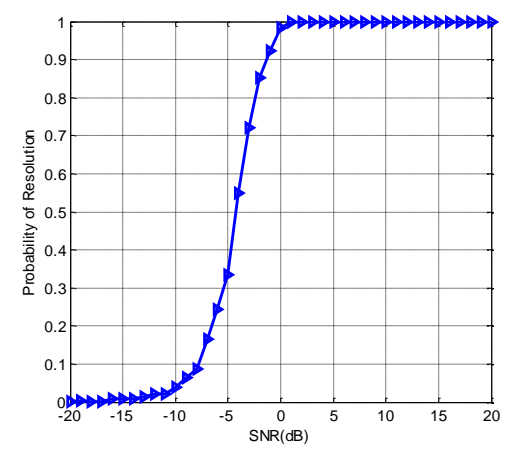

Fig. 4 Probability of resolution against the SNR

\section{Conclusions}

In this paper, we present a novel algorithm for DOA estimation based on the state-space balance method. Firstly, two hankel matrixes based on the received data are constructed. Then the singular value decomposition of the hankel matrix is used to obtain the signal subspace and singular value to estimate the DOA. This proposed algorithm acquires a good performance on the estimation error and a lower calculation compared with that of MUSIC algorithm while acquire a better performance on the estimation error and a quite efficiency calculation compared with that of ESPRIT algorithm. At the same time, the proposed method has the ability to estimate the DOAs of multi-targets by using only a single snapshot. Simulation studies show that the proposed algorithm is correct and useful. This method can be used in the high speed space targets to the real time processing.

\section{Acknowledgements}

This work was supported in part by the National Nature Science Foundation of China under grant 61471019, 61501011 and 61501012. 


\section{References}

[1] Kim H, Viberg M. Two decades of array signal processing research[J]. IEEE signal magazine, 1996, 13(4): 67-94.

[2] Xin J, Sano A. MSE-based regularization approach to direction estimation of coherent narrowband signals using linear prediction[J]. Signal Processing, IEEE Transactions on, 2001, 49(11): 2481-2497.

[3] Schmidt R O. Multiple emitter location and signal parameter estimation[J]. Antennas and Propagation, IEEE Transactions on, 1986, 34(3): 276-280.

[4] Stoica P, Nehorai A. Comparative performance study of element-space and beam-space MUSIC estimators[J]. Circuits, Systems and Signal Processing, 1991, 10(3): 285-292.

[5] Roy R, Kailath T. ESPRIT-estimation of signal parameters via rotational invariance techniques[J]. Acoustics, Speech and Signal Processing, IEEE Transactions on, 1989, 37(7): 984-995.

[6] Rao B D, Hari K V S. Performance analysis of ESPRIT and TAM in determining the direction of arrival of plane waves in noise[J]. Acoustics, Speech and Signal Processing, IEEE Transactions on, 1989, 37(12): 1990-1995.

[7] Viberg M, Ottersten B, Kailath T. Detection and estimation in sensor arrays using weighted subspace fitting[J]. Signal Processing, IEEE Transactions on, 1991, 39(11): 2436-2449.

[8] Ottersten B, Viberg M, Kailath T. Analysis of subspace fitting and ML techniques for parameter estimation from sensor array data[J]. Signal Processing, IEEE Transactions on, 1992, 40(3): 590-600.

[9] Yan F, Jin M, Qiao X. Low-complexity DOA estimation based on compressed MUSIC and its performance analysis[J]. IEEE transactions on signal processing, 2013, 61(5-8): 1915-1930.

[10] Wang J, Wei S M, Sun J P, et al. A GTD model and state space approach based method for extracting the UWB scattering center of moving target[J]. Science China Information Sciences, 2011, 54(1): 182-196.

[11] Wang Y L, Chen H, Peng Y N, et al. Spatial Spectrum Estimation Theory and Algorithm[M]. Tsinghua University Press Ltd, 2004.

[12] Akaike H. A new look at the statistical model identification[J]. Automatic Control, IEEE Transactions on, 1974, 19(6): 716-723.

[13] Wax M, Kailath T. Detection of signals by information theoretic criteria[J]. Acoustics, Speech and Signal Processing, IEEE Transactions on, 1985, 33(2): 387-392.

[14] Rissanen J. Modeling by shortest data description[J]. Automatica, 1978, 14(5): 465-471.

[15] Wax M, Ziskind I. Detection of the number of coherent signals by the MDL principle[J]. Acoustics, Speech and Signal Processing, IEEE Transactions on, 1989, 37(8): 1190-1196. 